\title{
Managing Local Order in Conjugated Polymer Blends via Polarity Contrast
}

\author{
Matthew J. Dyson, ${ }^{*,+, \neq}$ Eirini Lariou, ${ }^{\S}$ Jaime Martin, ${ }^{\|, \pi}$ Ruipeng Li, ${ }^{\#}$ Harikrishna \\ Erothu, ${ }^{\perp, \Delta}$ Guillaume Wantz, ${ }^{\diamond}$ Paul D. Topham, ${ }^{\perp}$ Olivier J. Dautel, ${ }^{\star}$ Sophia C. \\ Hayes, $*, \$$ \\ Paul N. Stavrinou, ${ }^{\nabla}$ and Natalie Stingelin*, ${ }^{*, \mathbf{m}, \circ}$
}

${ }^{\dagger}$ Molecular Materials and Nanosystems, Institute for Complex Molecular Systems, Eindhoven University of Technology, P.O. Box 513, 5600 MB Eindhoven, The Netherlands

${ }^{\ddagger}$ Department of Physics and Centre for Plastic Electronics, Imperial College London, Exhibition Road, London SW7 2AZ, U.K. § Department of Chemistry, University of Cyprus, P.O. Box 20537, 1678 Nicosia, Cyprus "POLYMAT,UniversityoftheBasqueCountryUPV/EHU,AvenidadeTolosa72,20018DonostiaSanSebastiań,Spain "Ikerbasque, Basque Foundation for Science, 48013 Bilbao, Spain

${ }^{\#}$ National Synchrotron Light Source II, Brookhaven National Laboratory, Upton, New York 11967, United States

${ }^{\perp}$ Aston Institute of Materials Research, Aston University, Birmingham B4 7ET, U.K.

${ }^{\Delta}$ Centre for Advanced Energy Studies (CAES), Koneru Lakshmaiah Education Foundation, Green Fields, Vaddeswaram, Guntur District, Andhra Pradesh-522 502, India

`Université de Bordeaux, IMS, CNRS, UMR-5218, Bordeaux INP, ENSCBP, 33405 Talence, France

Institut Charles Gerhardt de Montpellier, Laboratoire AM2N, UMR-5253, Université de Montpellier, ENSCM, CNRS, 8 rue de

${ }^{\nabla}$ Department of Engineering Science, University of Oxford, Parks Road, Oxford OX1 3PJ, U.K.

${ }^{\square}$ School of Materials Science \& Engineering and School of Chemical \& Biomolecular Engineering, Georgia Institute of Technology, Ferst Drive, Atlanta, Georgia 30332, United States

-Department of Materials and Centre for Plastic Electronics, Imperial College London, Exhibition Road, London SW7 2AZ, U.K.

${ }^{\circ}$ LaboratoiredeChimiedesPolymer̀esOrganiques-LCPO,UMR5629UniversitédeBordeaux,AlleéGeoffroySain tHilaire,

ABSTRACT: The optoelectronic landscape of conjugated polymers is intimately


related to their molecular arrangement and packing, with minute changes in local order, such as chain conformation and torsional backbone order/disorder, frequently having a substantial effect on macroscopic properties. While many of these local features can be manipulated via chemical design, the synthesis of a series of compounds is often required to elucidate correlations between chemical structure and macromolecular ordering. Here, we show that blending semiconducting polymers with insulating commodity plastics enables controlled manipulation of the semiconductor backbone planarity. The key is to create a polarity difference between the semiconductor backbone and its side chains, while matching the polarity of the side chains and the additive. We demonstrate the applicability of this approach through judicious comparison of regioregular poly(3hexylthiophene) (P3HT) with two of its more polar derivatives, namely the diblock copolymer poly(3hexylthiophene)-block-poly(ethylene oxide) (P3HT-b-PEO) and the graft polymer poly[3-but(ethylene oxide)thiophene] (P3BEOT), as well as their blends with poly(ethylene oxide) (PEO). Proximity between polar side chains and a similarly polar additive reduces steric hindrance between individual chain segments by essentially "expelling" the side chains away from the semiconducting backbones. This process, shown to be facilitated via exposure to polar environments such as humid air/water vapor, facilitates backbone realignment toward specific chain arrangements and, in particular, planar backbone configurations.

\section{INTRODUCTION}

In functional polymers, such as semiconducting plastics, chemical substitution with side chains is now a frequently used strategy to manipulate and control the local macro-

(c) 2019 American Chemical Society 6540

Special Issue: Jean-Luc Bredas Festschrift

Received: December 20, 2018 Revised: March 5, 2019 Published: March 6, 2019

DOI: 10.1021/acs.chemmater.8b05259 Chem. Mater. 2019, 31, 6540-6547

Downloaded via UNIV MONTPELLIER on December 14, 2020 at 14:52:47 (UTC).

See https://pubs.acs.org/sharingguidelines for options on how to legitimately share published articles.

Chemistry of Materials 
a

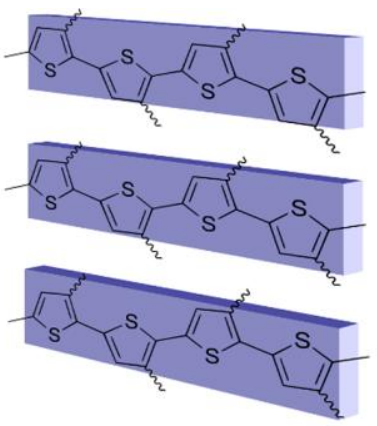

Neat $\left(20^{\circ} \mathrm{C}\right.$, dry atm)

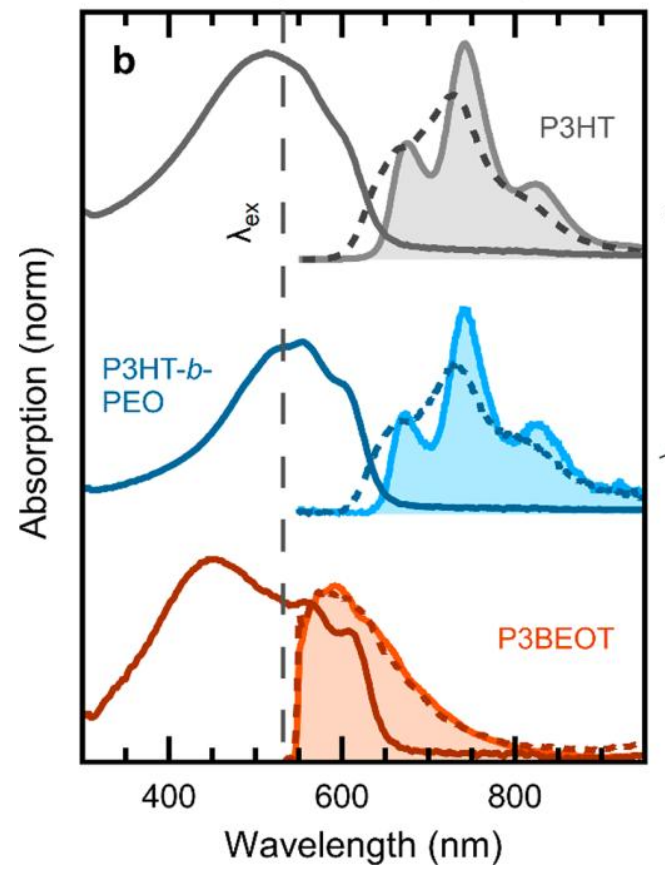

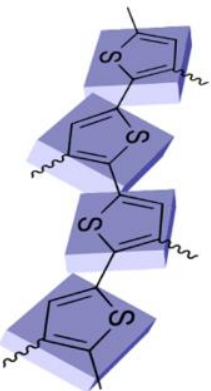

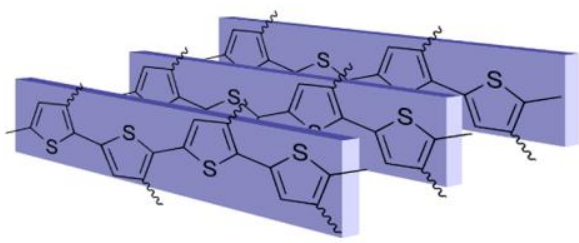

PEO Blend $\left(10^{\circ} \mathrm{C}\right.$, solvent atm $)$
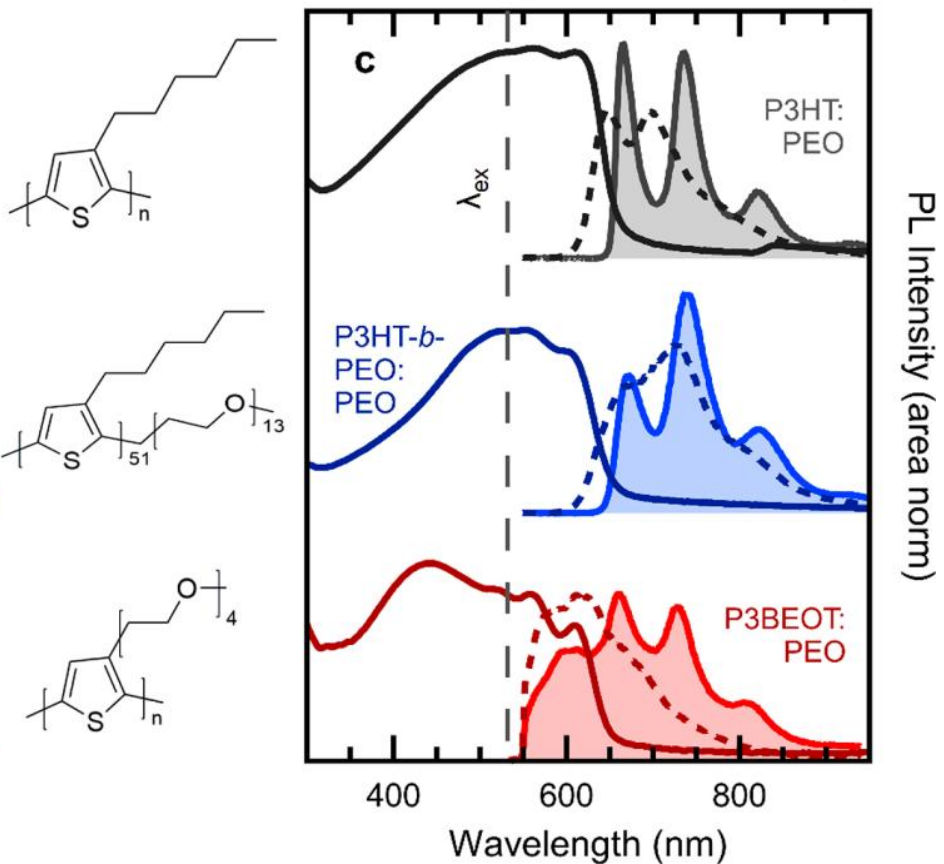

Figure 1. (a) Schematic showing three distinct types of local conjugated backbone order: lamellae (100) stacking (left), torsional disorder (center), and $\pi-\pi(010)$ stacking (right). Normalized absorption and photoluminescence (PL) spectra of (b) neat P3HT, P3HT-b-PEO, and P3BEOT cast from solution at $20^{\circ} \mathrm{C}$ and (c) $1: 1$ (weight percent) blends with PEO cast at $10^{\circ} \mathrm{C}$ and dried within a solvent-rich atmosphere. Absorption was determined at room temperature (solid line), and PL at room temperature (dashed) and $10 \mathrm{~K}$ (filled).

molecular arrangement and packing, including torsional back- bone order/disorder, lamellar stacking, and $\pi$-stacking (see

investigated, including blending with a second component, such

$1-4$

optoelectronic properties, such as charge-carrier transport ${ }^{5-7}$

Figure 1).

This can affect国often strongly国 contingent

elegant pathway to expand the functionality of the semi-

conductor, perhaps best exemplified by blends of prototypical 
poly(3-hexyl thiophene) (P3HT) with bulk insulating plastics such as poly(ethylene oxide) (PEO) that can be manipulated to display drastically different absorption behavior compared to the neat semiconductor. This difference was tentatively attributed to or absorption/emission features.

$8-10$

Originally, alkyl side

chains were introduced to aid solubility and, generally,

processability; ${ }^{11}$ more recently, polar side chains, such as those based on oligo(ethylene oxide) groups, have attracted interest as they render the base material more compatible with a change in the torsional backbone order of P3HT.

20

so-called "green" solvents.

12,13

Moreover, polymer semiconduc-

Here, we aim at combining these two approaches?chemical modification and blending?to control the molecular order of the active material and, thus, functionality. For this, we use model systems with the same conjugated backbone based on polythiophene chains? the P3HT homopolymer, the diblock tors, substituted with polar side chains, are interesting for bioelectronic applications, for instance, where mixed electron/ ion conduction is required (e.g., in electrochemical transistors used for biomimetic signal transduction, ion pumps, bioactive sensing elements), or where high biocompatibility is needed, $14-19$ 
copolymer poly(3-hexylthiophene)-block-poly(ethylene oxide) 25

including bio-integrated electronics and wearable devices. However, side chains can inhibit backbone packing and aggregation, and often introduce undesired torsional backbone disorder. Hence, alternative approaches to control structural (and other) features of polymer semiconductors have been

(P3HT-b-PEO), and the graft polymer poly[3-but(ethylene oxide)thiophene] (P3BEOT; for chemical structures see Figure 1b,c insets) ]and their blends with PEO. These judicious combinations of chemical structures and functionalities enable us to explore if blends can be designed from the outset such that

as a commodity plastic.

20-24

This has been shown to provide an

DOI: 10.1021/acs.chemmater.8b05259 Chem. Mater. 2019, 31, 6540-6547

Chemistry of Materials
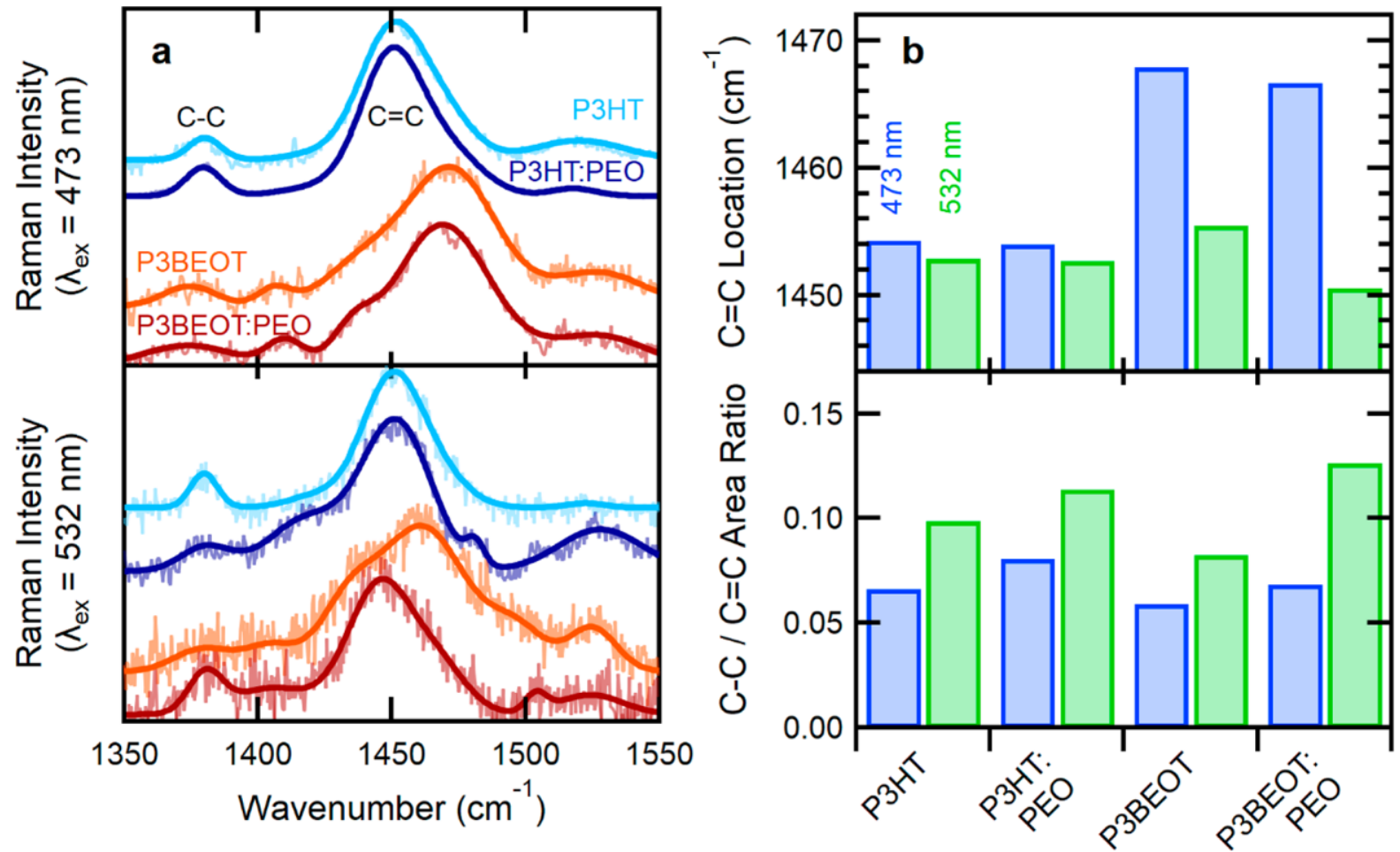

Figure 2. (a) Normalized resonance Raman spectra with $\lambda_{\mathrm{ex}}=473$ (top) and $532 \mathrm{~nm}$ (bottom) of P3HT, P3HT:PEO, P3BEOT, and P3BEOT:PEO, offset vertically for clarity. Original spectra are overlaid with fits (see Figures S4 and S5). (b) Extracted parameters for the symmetric C ?C stretching mode location (top) (an area weighted average of underlying peaks, higher values indicate increased torsional disorder) and C Q $\mathrm{C}$ to symmetric C?C peak area ratio (bottom) (higher values indicate greater planarity).

the active material's chain conformation and packing can be controlled by exploiting the different polarity-dependent compatibilities between certain moieties of the blend components. Indeed, the question we strive to answer is whether desirable macromolecular arrangements of polymeric semiconductors can be targeted by careful consideration of specific interactions between the semiconductor and the insulating polymer "additive". Utilizing the sensitivity of the optical properties of 
polythiophenes and their derivatives to backbone conformation ${ }^{26,27}$ thereby allows their local microstructure to be probed with a high degree of insight, providing a platform for future materials selection and processing criteria.

\section{RESULTS AND DISCUSSION}

We begin our discussion with the linear absorption and

photoluminescence (PL) spectra of thin films of the three neat polymers: $\mathrm{P} 3 \mathrm{HT}$ (molecular weight $\mathrm{M}_{\mathrm{w}}=$

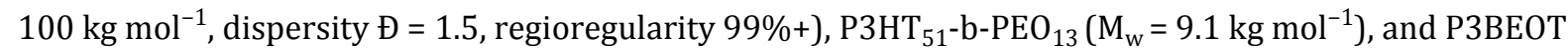
$\left(\mathrm{M}_{\mathrm{w}}=23 \mathrm{~kg} \mathrm{~mol}^{-1}, Ð=1.4\right.$, regioregularity 85\%+) (Figure 1b,c; films wirebar coated in air from $\mathrm{CHCl}_{3}$ solution with $10 \mathrm{mg} \mathrm{mL}^{-1}$ total polymer content). Immediately apparent from Figure $1 \mathrm{~b}$ is that the PEOblock in P3HT-b-PEO has only a small effect on the characteristic optical features of the P3HT moiety. The $0-0 / 0-1$ vibronic peak ratios in both absorption and emission, which are widely used sensitive indicators of the balance between intra- and interchain coupling, ${ }^{26,28-31}$ are essentially identical for P3HT and P3HTb-PEO. This is attributed to the P3HT segments in the block copolymer microphase separating from the PEO segments, ${ }^{32-35}$ leaving the former virtually unaffected by the latter and, thus, keeping the typical characteristics of P3HT. In stark contrast, the P3BEOT graft copolymer displays significant spectral differ-

6542

ences to the homopolymer, with only weak vibronic peaks observed in absorption at 560 and $610 \mathrm{~nm}$. The absorption maximum is found at $450 \mathrm{~nm}$. This blue shift relative to the P3HT homopolymer implies that P3BEOT comprises a larger fraction of non-aggregated, likely more torsionally disordered chain segments, similar to regiorandom P3HT (see Figure

S1).

36

Support for this view is provided by the PL spectra of the graft copolymers, recorded at room temperature and $10 \mathrm{~K}$, where we also observe a clear blue shift in spectral maxima, from $745 \mathrm{~nm}$ for the P3HT homopolymer to $595 \mathrm{~nm}$ for P3BEOT, accompanied by a clear loss of vibronic structure. Also noteworthy is the substantial overlap between absorption and emission spectra in P3BE0T, further reinforcing the picture that there is a significantly smaller fraction of torsionally ordered aggregates (with a smaller band gap) to which excitons can 
weight PEO (viscosity averaged molecular weight $M_{v}=900 \mathrm{~kg}$

$\mathrm{mol}^{-1}$ ), following the procedures reported in refs 20 and 38, we

find that adding PEO to the P3HT homopolymer leads to a

substantially enhanced $0-0 / 0-1$ peak ratio in both absorption

and emission, entirely consistent with earlier observations ${ }^{20}$ and

characteristic of a transition from inter- to intramolecular

coupling; ${ }^{26,29,31}$ similar changes in neat P3HT were previously

attributed to polar solvent induced planarization in solution

39,40

migrate prior to emission.

When blending the three polymers with high molecular

prior to aggregation.

In contrast, blending the block

copolymer P3HT-b-PEO with PEO has a negligible effect on

the optical response of this P3HT copolymer. We again assign

this observation to the tendency of block copolymers to

$32-35$

"additive" can be expected to form relatively phase-pure

In a blend, the PEO blocks and PEO microdomains, while the P3HT segments assemble in semi-

microphase separate.

DOI: 10.1021/acs.chemmater.8b05259 Chem. Mater. 2019, 31, 6540-6547

Chemistry of Materials

conductor-rich regions. This would drastically limit interactions of the PEO "additive" and/or PEO blocks with semiconducting chain segments, in agreement with our findings.

An entirely different scenario is observed for the P3BEOT:- PEO blend. For this system, we find the most significant change in optical behavior upon blending? especially in PL. Unlike the spectrum of the neat graft copolymer, the blend exhibits clear vibronic structure at $10 \mathrm{~K}$, although the emission spectrum characteristic of non-aggregated polythiophene segments is still dominant (subtracting this disordered component reveals a clear similarity with P3HT:PEO, albeit with a lower 0-0/0-1 peak ratio; see Figure S2). Furthermore, comparison of total luminescence spectra for P3BEOT and P3BEOT:PEO reveals a substantially higher emission intensity from the aggregated phase in the latter, relative to the non- 
aggregated phase (see Figure S3). Since this intensity variation is greater than the difference in absorption over the equivalent range of excitation wavelengths, this indicates a higher photoluminescent quantum yield in the blend, indicative of greater torsional order. Together, these changes imply that the high compatibility between the P3BEOT side chains and PEO via increased enthalpic interactions due to their mutual polarity leads to the inert component influencing the semiconducting backbone, affecting the latter's local assembly and hence its optoelectronic landscape. Notably, unlike the enhanced intramolecular coupling in P3HT:PEO, ${ }^{20}$ this process does not require a high-molecular-weight $\left(\mathrm{M}_{\mathrm{w}}>\right.$ entanglement threshold) semi- conducting component.

Based on the aforementioned findings, the P3BEOT system

was studied in greater detail, comparing it to P3HT and blends

of both with PEO. Focus was placed on changes related to local

ordering that could lead to the observed difference in the

absorption and emission spectra between the neat P3BEOT and

the P3BEOT:PEO binary blend, concentrating on torsional

backbone order as analyzed by resonance Raman spectroscopy.

This technique provides a sensitive probe of local molecular

arrangement and conformation in polymer semiconductors,

with selectivity achieved by exciting either non-aggregated or

aggregated fractions at 473 and $532 \mathrm{~nm}$, respectively. Figure 2a

shows experimental resonance Raman spectra overlaid with

$-1$

$\mathrm{cm}$. Taken together, these observations reinforce our

interpretation of the absorption spectra (Figure $1 b, c)$, specifically that the neat graft polymer is substantially more torsionally disordered than P3HT, particularly in the non- aggregated regions, with the aggregated regions comprising a certain fraction of segments of a similar backbone planarity to that found in P3HT. These regions are likely to be responsible for the weak vibronic structure shown in the UV-vis absorption spectrum of P3BEOT.

Moving on to the PEO binary blends, no significant effect on

the symmetric COC stretching peak location is found when

adding PEO to P3HT regardless of $\lambda_{\text {ex }}$, i.e., independent of

whether aggregated or non-aggregated regions are probed.

However, the COC/C C peak area ratio (see Figure 2b,

bottom panel) increases slightly for the blend compared to neat

P3HT, consistent with the addition of PEO slightly increasing 
P3HT backbone planarity. On the contrary, blending has a

striking effect on P3BEOT. Introduction of PEO leads to a

notable decrease in the symmetric CaC stretching mode

frequency of $\sim 5 \mathrm{~cm}^{-1}$ when probing aggregated regions (Figure

peak fits corresponding to the $\mathrm{C}$ ? $\mathrm{C}\left(\sim 1380 \mathrm{~cm}^{-1}\right)$, symmetric -1

C우 ( 1450-1470 cm ), and asymmetric CaC $(\sim 1525$

systems.

and for comparison P3HT, P3HT:PEO, and PEO, are shown in Figure S6, along with their scattered peak intensity along the out-of-plane $\left(\mathrm{Q}_{\mathrm{z}}\right)$ direction. The neat P3BEOT graft polymer lacks pronounced lamellar scattering peaks, typical for systems with limited long-range order; despite this, weak features are evident at low momentum transfer $\left(Q_{z} \leq 1 \AA^{-1}\right)$, indicating that a small proportion of chain segments display some propensity for translational order. Observations are very different for the blend, with P3BEOT:PEO showing a series of intense scattering peaks at low momentum transfer that cannot be attributed to PEO. Three scattering peaks can be seen at $\mathrm{Q}_{\mathrm{z}}=0.24,0.48$, and $0.72 \AA^{-1}$, with an interval of $\sim 0.24 \AA^{-1}$, which we assign to the (100), (200), and (300) diffractions of the semiconductor. Accordingly, we infer a lamellar stacking distance of $\sim 2.61 \mathrm{~nm}$. This is larger than for the P3HT homopolymer, neat or blended with PEO, where the (100) diffraction is found at $Q_{z}=0.39 \AA^{-1}$ (lamellae stacking distance $\sim 1.61 \mathrm{~nm}$ ), and can be attributed to the longer side chains of P3BEOT compared to P3HT. Indeed, this distance is close to those of poly(alkyl thiophene)s with similar side-chain lengths to P3BEOT, such as poly(3-dodecyl thiophene) (P3DDT), with a $Q_{z}$ peak at $\sim 0.25 \AA^{-1}$ (lamellae

DOI: 10.1021/acs.chemmater.8b05259 Chem. Mater. 2019, 31, 6540-6547

$\mathrm{cm}^{-1}$ ) stretching modes (see Figures S4 and S5 for fitting and

residuals following excitation at 473 and $532 \mathrm{~nm}$, respec-

Scattering patterns for P3BEOT and P3BEOT:PEO,

tively).

41,42

Increased intensity of the $\mathrm{C}$ ? $\mathrm{C}$ stretch relative to

the dominant symmetric C?C mode, and a shift of the

symmetric CQC band to lower frequencies, have previously

been correlated with increased planarity between monomer

units due to enhanced $\pi$-electron delocalization.

41,42

Therefore,

the frequency of the symmetric $\mathrm{C}$ ? $\mathrm{C}$ band and the $\mathrm{C}$ ? $\mathrm{C} / \mathrm{C}$ ? 
C intensity ratio was followed to assess the torsional order of the

polythiophene backbones in our systems, expecting C?C

stretching vibrations for torsionally disordered chains to be

around $1470 \mathrm{~cm}^{-1}$ and those for planar chains around 1450

$-1$

. Turning attention to the neat materials (i.e., P3BEOT and P3HT), when exciting their non-aggregated regions $\left(\lambda_{\mathrm{ex}}=473 \mathrm{~nm}\right)$, the symmetric C $\mathrm{C}$. stretching band (an area-weighted average of two underlying peaks) appears at $\sim 1465$ and $\sim 1454 \mathrm{~cm}^{-1}$ for P3BEOT and P3HT, respectively (see Figure $2 \mathrm{~b}$, top panel; fits are presented in Figures S1 and S2). Probing more aggregated domains $\left(\lambda_{\mathrm{ex}}=532 \mathrm{~nm}\right)$, the symmetric C ? $\mathrm{C}$ stretching mode was observed at $\sim 1456 \mathrm{~cm}^{-1}$ for P3BEOT, while in P3HT this mode remains almost unchanged at $\sim 1453$

6543

$\mathrm{cm}$

$2 \mathrm{~b}$, top panel), resulting in a peak at $\sim 1451 \mathrm{~cm}$

$-1$

. This frequency

is slightly $\left(\sim 2 \mathrm{~cm}^{-1}\right)$ lower than found for neat P3HT and its

blends with PEO, indicating that the addition of PEO to the graft

polymer substantially increases the fraction of chain segments

with increased backbone planarity, especially in aggregated

regions, often beyond that achievable in neat or blended P3HT.

This conclusion is supported by the $\mathrm{C}$ ? $\mathrm{C} / \mathrm{C}$ ? $\mathrm{C}$ peak area

ratio on probing aggregated regions $\left(\lambda_{\mathrm{ex}}=532 \mathrm{~nm}\right)$; for

P3BEOT:PEO, it is somewhat higher than for both P3HT and

41,42

exciting the aggregated fraction.

Since the increased backbone planarity does not necessarily

imply increased aggregation or even long-range order (but will

play a role in determining the balance between inter- and

intrachain coupling within aggregates), ${ }^{26,31}$ grazing-incidence

wide-angle X-ray scattering (GIWAXS) was performed to probe 
the crystalline ordering and orientation in the polymer

43

In contrast, blending has a far less pronounced effect on the non-aggregated fraction $\left(\lambda_{\mathrm{ex}}=473 \mathrm{~nm}\right)$, with the reduction in symmetric $\mathrm{C}$. $\mathrm{C}$ location being comparatively negligible at $\sim 1 \mathrm{~cm}^{-1}$, and a smaller increase in $\mathrm{C}$ ? $\mathrm{C} / \mathrm{C}$ 目 $\mathrm{C}$ peak ratio found than when

P3HT:PEO (Figure 2b, bottom panel).

Chemistry of Materials

stacking distance $\sim 2.51 \mathrm{~nm})^{44}$ ?although the addition of PEO may also contribute to the observed effect. Our observations clearly show that, in addition to affecting backbone planarity and smaller-scale aggregation, blending P3BEOT with PEO induces long-range packing that can be revealed by X-ray scattering? specifically, clear orientational order with P3BEOT lamellae stacking predominantly out-ofplane ((h00) peaks are only found along $\mathrm{Q}_{\mathrm{z}}$ ).

\section{CONCLUSIONS}

Blending with insulating polymers can be an efficient tool to manipulate the local, and to a certain extent long-range, arrangement of polymeric semiconductors. Microphase separa- tion, as found in block copolymers (neat and in blends), has been shown to have little effect on local molecular ordering, whereas considerable structural changes can be induced in blends with graft polymers as the active component. For this to occur, it appears that two criteria must be satisfied: (i) there must be a strong contrast in polarity between the semiconductor backbone and its side chains, and (ii) there must be a strong affinity between the semiconductor side chains and the insulating polymer "additive". Such a scenario creates an enthalpic driving force that "draws" the polar side chains away from the active material's backbone toward the second blend component, reducing the side-chain disorder and, possibly, the steric hindrance that normally would lead to torsional disorder and reduced aggregation, especially in materials with relatively bulky side chains. These enthalpic interactions overcome the plasticizing effect of PEO, which lowers the fictive temperature (an experimentally accessible substitute for the glass transition temperature, $\left.\mathrm{T}_{\mathrm{g}}\right)^{45}$ of P3BEOT in the blends by $\sim 9^{\circ} \mathrm{C}$ compared to the neat system (see Figure S7; with additional data for neat P3BEOT shown in Figure S8). In the absence of a polarity contrast, such a depression of the glass transition temperature leads to more rapid segmental backbone relaxation, which in turn can be expected to lead to increased torsional backbone disorder. In contrast, in the case of highpolarity blends, the increased backbone mobility may assist in adopting a more planar backbone conformation, facilitating backbone re- arrangement.

The polarity contrast between backbone and side chains/

additive can, of course, be manipulated in a rather straightfor-

ward fashion. For instance, herein insulating polymer "additives"

were selected that are structurally similar to PEO but have

different Hansen solubility parameters and, therefore, have

different interactions with the P3BEOT side chains]i.e., a 

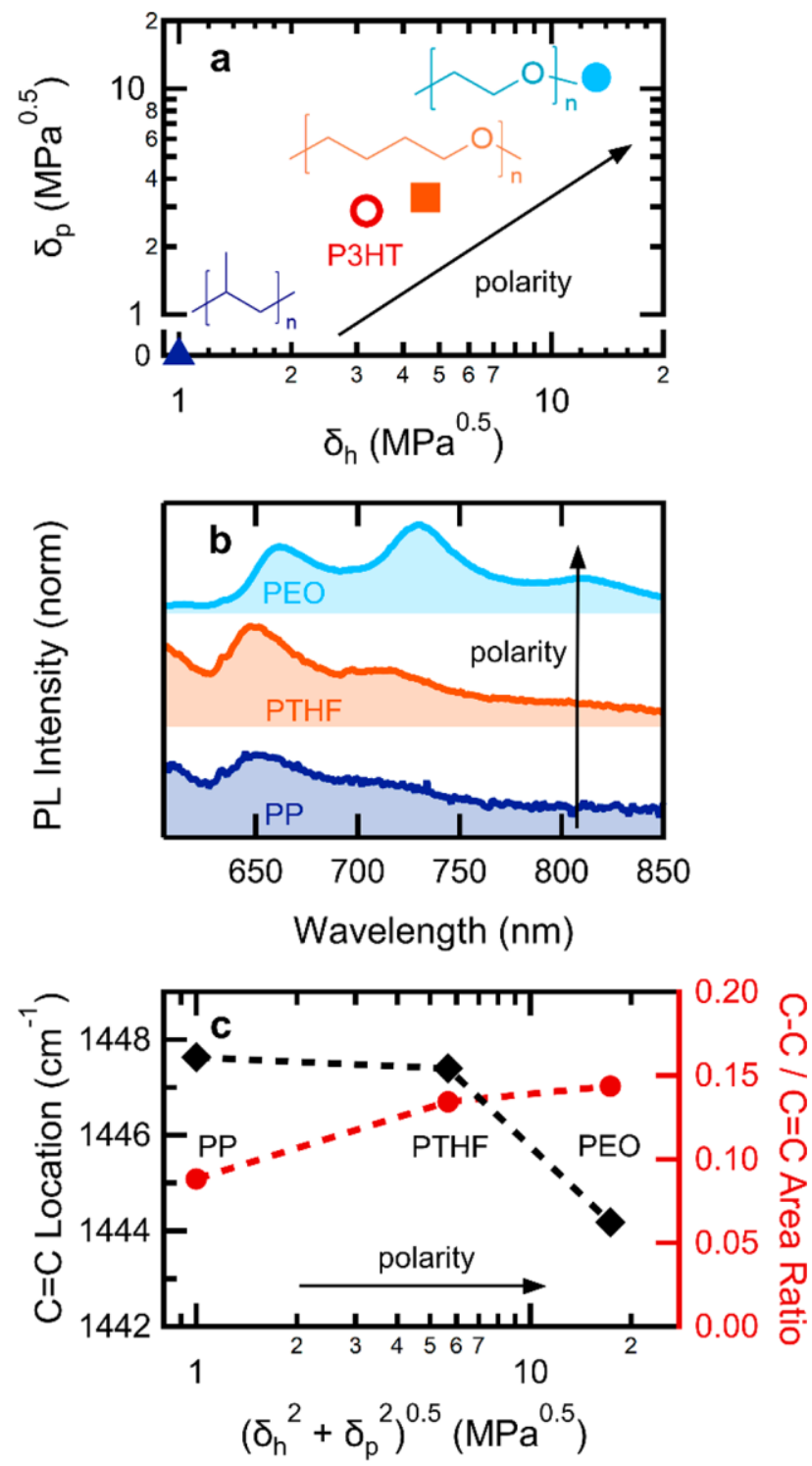

different polarity contrast (Figure 3a and SI).

increasing the polarity of the "additive polymer" leads to more pronounced vibronic structures in the PL of the blend when probing aggregated species $\left(\lambda_{\mathrm{ex}}=580 \mathrm{~nm}\right.$; Figure $\left.3 \mathrm{~b}\right)$, and to a lower-frequency symmetric $\mathrm{C}$ 国 band and correspondingly higher $\mathrm{C}$ [ $\mathrm{C} / \mathrm{C}$ 回 $\mathrm{C}$ peak ratio in resonant Raman spectroscopy $\left(\lambda_{\mathrm{ex}}=532 \mathrm{~nm}\right.$; Figure 3c, spectra in Figure S9). These findings substantiate our interpretation that interactions between polar side chains and a similarly polar insulating "additive" promote planarization of the semiconducting backbone by drawing away side chains and, thus, reducing steric hindrance. Polarity contrast is clearly critical. Examples of blends with strong affinity between the side chain and additive but with no polarity contrast between side chains/additive and the conjugated backbone of the main component, such as binary blends of poly[3-(2'-ethyl)- hexylthiophene $]$ (P3EHT) ${ }^{50}$ comprising a poly(thiophene) backbone with branched alkyl side chains, and ultra-low-density

6544

Figure 3. (a) Insulating polymers with different polar $\delta_{\mathrm{p}}$ and hydrogen- bonding $\delta_{\mathrm{h}}$ Hansen solubility parameters. (b) $10 \mathrm{~K}$ PL of P3BEOT:PEO, P3BEOT:PTHF, and P3BEOT:PP $\left(\lambda_{\mathrm{ex}}=580 \mathrm{~nm}\right)$ shows an increase in vibronic structure with insulator polarity. (c) Resonance Raman symmetric C Q $\mathrm{C}$ peak location decreases and C. $\mathrm{C} / \mathrm{C}$. $\mathrm{C}$ peak area ratio $\left(\lambda_{\mathrm{ex}}=\right.$ $532 \mathrm{~nm}$ ) increases with insulator polarity (distance from origin in (a)), indicating reduced torsional disorder (spectra, fits, and residuals in Figure S9). 
polyethylene (ULDPE), a highly branched version of poly- ethylene, show that blending has little effect on local order of the semiconductor (Figure S10).

It should be noted that the polarity of the local environment can also be affected by small-molecular additives. This can be exemplified by experiments involving the controlled exposure of neat P3BEOT and its blend with PEO to water vapor. Water will increase the polarity contrast between backbone and side chains/PEO; it also will act as a plasticizer, ${ }^{39,51}$ lowering the $\mathrm{T}_{\mathrm{g}}$ of the neat P3BEOT and P3BEOT:PEO, as confirmed by fast differential scanning calorimetry measurements (DSC, Figure S11). Intuitively, one would assume plasticization should increase the torsional backbone disorder of P3BEOT (as discussed above) leading to less electronic coupling between semiconducting chains and, hence, weaker vibronic structure in

DOI: 10.1021/acs.chemmater.8b05259 Chem. Mater. 2019, 31, 6540-6547

Reassuringly,

Chemistry of Materials

UV-vis absorption and PL spectra. However, after incorporat- ing water into P3BEOT and P3BEOT:PEO blends via exposure to water vapor, both systems display a more pronounced vibronic structure both in absorption and PL (see Figure S12), from which we infer that also this treatment leads to higher torsional backbone order, with the effect being notably stronger for the blends.

The effect of small-molecular additives is, however, not necessarily stable. This is reflected by the structural evolution of P3BEOT and its blends with PEO directly after casting and drying la process where humidity is trapped in the resulting thin films?and after aging over a period of 4 months in ambient conditions, as followed by resonance Raman spectroscopy. For the neat graft copolymer, when exciting aggregated regions $\left(\lambda_{\mathrm{ex}}=532 \mathrm{~nm}\right)$, the frequency of its symmetric CaC stretching mode initially is, intriguingly, very low $\left(\sim 1447 \mathrm{~cm}^{-1}\right)$ and comparable to that of P3BEOT:PEO $\left(\sim 1444 \mathrm{~cm}^{-1}\right.$, Figure S13; fits shown in Figure S14). This implies that, initially, the neat graft copolymer comprises a high fraction of planar chain segments. However, this dominant mode shifts over time to higher frequencies until "saturating" after 4 months at $\sim 1456 \mathrm{~cm}^{-1}$, accompanied by a noticeable reduction in CQOC/C国 peak area ratio. In stark contrast, the polymer blend is markedly more stable over time with respect to structure. A change in peak frequency in the blend is observed over time, but one that is drastically smaller than that observed for the neat graft polymer, with aged samples featuring a symmetric $\mathrm{C}$ Q $\mathrm{C}$ stretching mode at

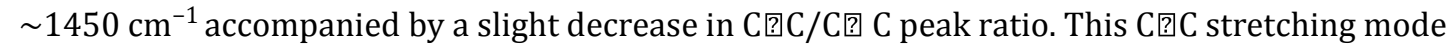
frequency is still slightly smaller than what is found for neat P3HT, while the $\mathrm{C}$ 目 $\mathrm{C} / \mathrm{C}$ ? $\mathrm{C}$ peak ratio is higher, which shows that in P3BEOT:PEO blends, the P3BEOT backbone segments remain more planar than in P3HT and P3HT:PEO binary blends, also when aged for 4 months (Figure S13b). We attribute the instability of the planarization effect of water/humidity in neat P3BEOT to the relatively rapid release of water from this system. In contrast, water molecules retained in the P3BEOT:PEO binary blends due to the hydrophilic $\mathrm{PEO}^{52,53}$ stay trapped, contributing to the polarity contrast and assisting in planarizing the semiconductor backbone. This is supported by UV-vis and PL spectroscopy (see SI, Figure S12) and highlights the benefit of blending with polymeric matrices.

The moisture dependence of P3BEOT and its blends with PEO further emphasizes the strong correlation between local environment and backbone ordering (and consequently, optical response), and hence the wide applicability via selection of side- chain polarity, polarity of blend component, as well as smallmolecular additives, such as water, that further affect the polarity contrast between backbone and environment. Our findings, thus, open a simple alternative pathway to structural control without the need to realize all functionality exclusively via (often complex) chemical design. This approach will be particularly important in fields where materials comprising bulky side chains are usedofor instance, bioelectronics where more polar moieties are frequently added to the active material to increase 
biocompatibility, increase water-induced swelling, and enhance ion flow. In these scenarios, blending should allow for control of local ordering and packing, while side-chain substitution can be used to introduce other desired functionalities.

\title{
ASSOCIATED CONTENT
}

\author{
* Supporting Information \\ The Supporting Information is available free of charge on the ACS Publications website at DOI: \\ 10.1021/acs.chemma- ter.8b05259.
}

Experimental methods; further discussion of GIWAXS, solubility, and humidity; further analysis of absorption and PL spectra; site-selective PL and total luminescence spectra; additional Raman spectra and fitting; fast-DSC scans and analysis; GIWAXS measurements; humidity- dependent absorption and PL spectra; synthetic methods; and NMR spectra, including Figures S1-S19 (PDF)

\section{AUTHOR INFORMATION}

\author{
Corresponding Authors \\ *E-mail (M.J.D.): m.j.dyson@tue.nl. \\ *E-mail (S.C.H.): shayes@ucy.ac.cy. \\ *E-mail (N.S.): natalie.stingelin@mse.gatech.edu.
}

ORCID

Matthew J. Dyson: 0000-0002-4502-6250 Jaime Martin: 0000-0002-9669-7273

Paul D. Topham: 0000-0003-4152-6976 Olivier J. Dautel: 0000-0003-2327-5376 Sophia C. Hayes: 00000003-0238-6915 Paul N. Stavrinou: 0000-0001-6075-2587 Natalie Stingelin: 0000-0002-1414-4545 Notes

The authors declare no competing financial interest.

ACKNOWLEDGMENTS

M.J.D., P.N.S., and N.S. thank the UK's Engineering and Physical Sciences Research Council (EPSRC) for funding via the Centre for Doctoral Training in Plastic Electronics Materials (EP/G037515/1). M.J.D., N.S., P.N.S., S.C.H., and E.L. also thank the Marie Skłodowska-Curie Actions Innovative Training Network "H2020-MSCAITN-2014 INFORM-675867". J.M. furthermore thanks MINECO for the Ramon y Cajal contract and the Ikerbasque Foundation for the Ikerbasque Research Fellow program, and N.S. gratefully acknowledges additional support from the IdEx Bordeaux Excellence program (ANR-10- IDEX-03-02). P.D.T. and H.E. acknowledge funding from the European Union Seventh Framework Program (FP7/2010 SYNABCO no. 273316 and FP7/2011 under grant agreement ESTABLIS no. 290022). This work is based upon research conducted at the Cornell High Energy Synchrotron Source (CHESS), which is supported by the National Science Foundation under award DMR-1332208. The authors thank, in addition, Prof. Carlos Silva (Department of Physics, Georgia Institute of Technology, Atlanta, Georgia, USA) and Prof. Iain McCulloch (Physical Sciences and Engineering Division, King Abdullah University of Science and Technology (KAUST), Saudi Arabia) for many helpful discussions. 
(1) Mei, J.; Bao, Z. Side Chain Engineering in Solution-Processable Conjugated Polymers. Chem. Mater. 2014, 26, 604-615.

(2) Himmelberger, S.; Duong, D. T.; Northrup, J. E.; Rivnay, J.; Koch, F. P. V.; Beckingham, B. S.; Stingelin, N.; Segalman, R. A.; Mannsfeld, S. C. B.; Salleo, A. Role of Side-Chain Branching on Thin-Film Structure and Electronic Properties of Polythiophenes. Adv. Funct. Mater. 2015, 25, 2616-2624.

DOI: 10.1021/acs.chemmater.8b05259 Chem. Mater. 2019, 31, 6540-6547

Chemistry of Materials

(3) Duan, C.; Willems, R. E. M.; van Franeker, J. J.; Bruijnaers, B. J.; Wienk, M. M.; Janssen, R. A. J. Effect of Side Chain Length on the Charge Transport, Morphology, and Photovoltaic Performance of Conjugated Polymers in Bulk Heterojunction Solar Cells. J. Mater. Chem. A 2016, 4, 1855-1866.

(4) Kim, J.-H.; Wood, S.; Park, J. B.; Wade, J.; Song, M.; Yoon, S. C.; Jung, I. H.; Kim, J.-S.; Hwang, D.-H. Optimization and Analysis of Conjugated Polymer Side Chains for High-Performance Organic Photovoltaic Cells. Adv. Funct. Mater. 2016, $26,1517-1525$.

(5) Kumar, A.; Baklar, M. A.; Scott, K.; Kreouzis, T.; Stingelin- Stutzmann, N. Efficient, Stable Bulk Charge Transport in Crystalline/ Crystalline Semiconductor-Insulator Blends. Adv. Mater. 2009, 21, 4447-4451.

(6) Han, S.; Yu, X.; Shi, W.; Zhuang, X.; Yu, J. Solvent-Dependent Electrical Properties Improvement of Organic FieldEffect Transistor Based on Disordered Conjugated Polymer/Insulator Blends. Org. Electron. 2015, 27, 160-166.

(7) Ong, B. S.; Wu, Y.; Liu, P.; Gardner, S. High-Performance Semiconducting Polythiophenes for Organic Thin-Film Transistors. J. Am. Chem. Soc. 2004, 126, 3378-3379.

(8) Delongchamp, D. M.; Kline, R. J.; Fischer, D. A.; Richter, L. J.; Toney, M. F. Molecular Characterization of Organic Electronic Films. Adv. Mater. 2011, 23, 319-337.

(9) Botiz, I.; Astilean, S.; Stingelin, N. Altering the Emission Properties of Conjugated Polymers. Polym. Int. 2016, 65, 157-163.

(10) Raithel, D.; Simine, L.; Pickel, S.; Schötz, K.; Panzer, F.; Baderschneider, S.; Schiefer, D.; Lohwasser, R.; Köhler, J.; Thelakkat, M.; et al. Direct Observation of Backbone Planarization via Side-Chain Alignment in Single BulkySubstituted Polythiophenes. Proc. Natl. Acad. Sci. U. S. A. 2018, 115, 2699-2704.

(11) Roncali, J. Conjugated Poly(Thiophenes): Synthesis, Function- alization, and Applications. Chem. Rev. 1992, 92, 711-738.

(12) Chen, X.; Zhang, Z.; Ding, Z.; Liu, J.; Wang, L. Diketopyrrolopyrrole-Based Conjugated Polymers Bearing Branched Oligo(Ethylene Glycol) Side Chains for Photovoltaic Devices. Angew. Chem., Int. Ed. 2016, 55, 10376-10380.

(13) Lee, C.; Lee, H. R.; Choi, J.; Kim, Y.; Nguyen, T. L.; Lee, W.; Gautam, B.; Liu, X.; Zhang, K.; Huang, F.; et al. Efficient and Air-Stable Aqueous-Processed Organic Solar Cells and Transistors: Impact of Water Addition on Processability and Thin-Film Morphologies of Electroactive Materials. Adv. Energy Mater. 2018, 8, 1802674.

(14) Giovannitti, A.; Sbircea, D.-T.; Inal, S.; Nielsen, C. B.; Bandiello, E.; Hanifi, D. A.; Sessolo, M.; Malliaras, G. G.; McCulloch, I.; Rivnay, J. Controlling the Mode of Operation of Organic Transistors through Side-Chain Engineering. Proc. Natl. Acad. Sci. U. S. A. 2016, 113, 12017-12022.

(15) Pacheco-Moreno, C. M.; Schreck, M.; Scaccabarozzi, A. D.; Bourgun, P.; Wantz, G.; Stevens, M. M.; Dautel, O. J.; Stingelin, N.; et al. The Importance of Materials Design to Make Ions Flow : Toward Novel Materials Platforms for Bioelectronics Applications. Adv. Mater. 2017, 29, 1604446.

(16) Rivnay, J.; Owens, R. M. R. M.; Malliaras, G. G. The Rise of Organic Bioelectronics. Chem. Mater. 2014, 26, 679-685. 
(17) Simon, D. T.; Gabrielsson, E. O.; Tybrandt, K.; Berggren, M. Organic Bioelectronics: Bridging the Signaling Gap between Biology and Technology. Chem. Rev. 2016, 116, 13009-13041.

(18) Nielsen, C. B.; Giovannitti, A.; Sbircea, D.; Bandiello, E.; Niazi, M. R.; Hanifi, D. A.; Sessolo, M.; Amassian, A.; Malliaras, G. G.; Rivnay, J.; et al. Molecular Design of Semiconducting Polymers for High- Performance Organic Electrochemical Transistors. J. Am. Chem. Soc. 2016, 138, 10252-10259.

(19) Giovannitti, A.; Nielsen, C. B.; Sbircea, D. T.; Inal, S.; Donahue, M.; Niazi, M. R.; Hanifi, D. A.; Amassian, A.; Malliaras, G. G.; Rivnay, J.; McCulloch, I. N-Type Organic Electrochemical Transistors with Stability in Water. Nat. Commun. 2016, 7,13066

(20)Hellmann,C.;Paquin,F.;Treat,N.D.;Bruno,A.;Reynolds,L. X.; Haque, S. A.; Stavrinou, P. N.; Silva, C.; Stingelin, N. Controlling the Interaction of Light with Polymer Semiconductors. Adv. Mater. 2013, 25, 4906-4911.

6546

(21) Scaccabarozzi, A. D.; Stingelin, N. Semiconducting:Insulating Polymer Blends for Optoelectronic Applications?a Review of Recent Advances. J. Mater. Chem. A 2014, 2, 10818-10824.

(22) Jahnke, A. A.; Yu, L.; Coombs, N.; Scaccabarozzi, A. D.; Tilley, A. J.; Dicarmine, P. M.; Amassian, A.; Stingelin, N.; Seferos, D. S. Polytellurophenes Provide Imaging Contrast towards Unravelling the Structure-Property-Function Relationships in Semiconductor: Insu- lator Polymer Blends. J. Mater. Chem. C 2015, 3, 3767-3773.

(23) Strobel, N.; Eckstein, R.; Lehr, J.; Lemmer, U.; Hernandez-Sosa, G. Semiconductor:Insulator Blends for Speed Enhancement in Organic Photodiodes. Adv. Electron. Mater. 2018, 4, 1700345.

(24) Abbaszadeh, D.; Kunz, A.; Wetzelaer, G. A. H. H.; Michels, J. J.; Craćiun,N.I.;Koynov,K.;Lieberwirth,I.;Blom,P.W.M.M. Elimination of Charge Carrier Trapping in Diluted Semiconductors. Nat. Mater. 2016, 15, 628-633.

(25) Erothu, H.; Sohdi, A. A.; Kumar, A. C.; Sutherland, A. J.; Dagron- Lartigau, C.; Allal, A.; Hiorns, R. C.; Topham, P. D. Facile Synthesis of Poly(3-Hexylthiophene)-Block-Poly(Ethylene Oxide) Copolymers via Steglich Esterification. Polym. Chem. 2013, 4, 3652-3655.

(26) Niles, E. T.; Roehling, J. D.; Yamagata, H.; Wise, A. J.; Spano, F. C.; Moule', A. J.; Grey, J. K. J-Aggregate Behavior in Poly-3- Hexylthiophene Nanofibers. J. Phys. Chem. Lett. 2012, 3, 259-263.

(27)Greǵoire,P.;Vella,E.;Dyson,M.;Bazań,C.M.;Leonelli,R.; Stingelin, N.; Stavrinou, P. N.; Bittner, E. R.; Silva, C.; et al. Excitonic Coupling Dominates the Homogeneous Photoluminescence Excitation Linewidth in Semicrystalline Polymeric Semiconductors. Phys. Rev. B: Condens. Matter Mater. Phys. 2017, 95, 180201.

(28) Clark, J.; Silva, C.; Friend, R.; Spano, F. C. Role of Intermolecular Coupling in the Photophysics of Disordered Organic Semiconductors: Aggregate Emission in Regioregular Polythiophene. Phys. Rev. Lett. 2007, 98, 206406.

(29)Paquin,F.;Yamagata,H.;Hestand,N.J.;Sakowicz,M.;Beŕube', N.; Côte', M.; Reynolds, L. X.; Haque, S. A.; Stingelin, N.; Spano, F. C.; et al. Two-Dimensional Spatial Coherence of Excitons in Semicrystal- line Polymeric Semiconductors: Effect of Molecular Weight. Phys. Rev. B: Condens. Matter Mater. Phys. 2013, 88, 155202.

(30) Yamagata, H.; Spano, F. C. Interplay between Intrachain and Interchain Interactions in Semiconducting Polymer Assemblies: The HJ-Aggregate Model. J. Chem. Phys. 2012, 136, 184901.

(31) Spano, F. C.; Silva, C. H- and J-Aggregate Behavior in Polymeric Semiconductors. Annu. Rev. Phys. Chem. 2014, 65, $477-500$

(32) Patel, S. N.; Javier, A. E.; Beers, K. M.; Pople, J. A.; Ho, V.; Segalman, R. A.; Balsara, N. P. Morphology and Thermodynamic Properties of a Copolymer with an Electronically Conducting Block: Poly(3-Ethylhexylthiophene)Block-Poly(Ethylene Oxide). Nano Lett. 2012, 12, 4901-4906.

(33) Park, S.; Kang, S.; Fryd, M.; Saven, J. G.; Park, S. Highly Tunable Photoluminescent Properties of Amphiphilic Conjugated Block- Copolymers Highly Tunable Photoluminescent Properties of Amphi- philic Conjugated BlockCopolymers. J. Am. Chem. Soc. 2010, 132, 9931-9933. 
(34) Erothu, H.; Kolomanska, J.; Johnston, P.; Schumann, S.; Deribew, D.; Toolan, D. T. W.; Gregori, A.; Dagron-Lartigau, C.; Portale, G.; Bras, W.; et al. Synthesis, Thermal Processing, and Thin Film Morphology of Poly(3-Hexylthiophene)-

Poly(Styrenesulfonate) Block Copolymers. Macromolecules 2015, 48, 2107-2117.

(35) Topham, P. D.; Parnell, A. J.; Hiorns, R. C. Block Copolymer Strategies for Solar Cell Technology. J. Polym. Sci., Part B: Polym. Phys.

$2011,49,1131-1156$.

(36) Korovyanko, O.; Osterbacka, R.; Jiang, X.; Vardeny, Z.; Janssen,

R. Photoexcitation Dynamics in Regioregular and Regiorandom Polythiophene Films. Phys. Rev. B: Condens. Matter Mater. Phys. 2001, 64, 235122.

(37)Hoffmann,S.T.;Bas̈sler,H.;Koenen,J.M.;Forster,M.;Scherf, U.; Scheler, E.; Strohriegl, P.; Köhler, A. Spectral Diffusion in Poly(Para-Phenylene)-Type Polymers with Different Energetic Dis- order. Phys. Rev. B: Condens. Matter Mater. Phys. 2010, 81, 115103.

DOI: 10.1021/acs.chemmater.8b05259 Chem. Mater. 2019, 31, 6540-6547

Chemistry of Materials

(38) Zhao, K.; Yu, X.; Li, R.; Amassian, A.; Han, Y. Solvent-Dependent Self-Assembly and Ordering in Slow-Drying DropCast Conjugated Polymer Films. J. Mater. Chem. C 2015, 3, 9842-9848.

(39) Scharsich, C.; Lohwasser, R. H.; Sommer, M.; Asawapirom, U.; Scherf, U.; Thelakkat, M.; Neher, D.; Köhler, A. Control of Aggregate Formation in Poly(3-Hexylthiophene) by Solvent, Molecular Weight, and Synthetic Method. J. Polym. Sci., Part B: Polym. Phys. 2012, 50, 442-453.

(40)Panzer,F.;Bas̈sler,H.;Köhler,A.TemperatureInducedOrder- Disorder Transition in Solutions of Conjugated Polymers Probed by Optical Spectroscopy. J. Phys. Chem. Lett. 2017, 8, 114-125.

(41) Gao, Y.; Grey, J. K. Resonance Chemical Imaging of Polythiophene/Fullerene Photovoltaic Thin Films: Mapping

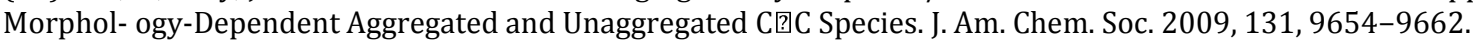

(42) Tsoi, W. C.; James, D. T.; Kim, J. S.; Nicholson, P. G.; Murphy, C. E.; Bradley, D. D. C.; Nelson, J.; Kim, J. S. The Nature of In-Plane Skeleton Raman Modes of P3HT and Their Correlation to the Degree of Molecular Order in P3HT:PCBM Blend Thin Films. J. Am. Chem. Soc. 2011, 133, 9834-9843.

(43) Rivnay, J.; Mannsfeld, S. C. B.; Miller, C. E.; Salleo, A.; Toney, M. F. Quantitative Determination of Organic Semiconductor Micro- structure from the Molecular to Device Scale. Chem. Rev. 2012, 112, 5488-5519.

(44) Sauve', G.; Javier, A. E.; Zhang, R.; Liu, J.; Sydlik, S. A.; Kowalewski, T.; McCullough, R. D. Well-Defined, High Molecular Weight Poly(3-Alkylthiophene)s in Thin-Film Transistors: Side Chain Invariance in Field-Effect Mobility. J. Mater. Chem. 2010, 20, 3195- 3201.

(45) Badrinarayanan, P.; Zheng, W.; Li, Q.; Simon, S. L. The Glass Transition Temperature versus the Fictive Temperature. J. Non-Cryst. Solids 2007, 353, 2603-2612.

(46) Barton, A. F. M. Solubility Parameters. Chem. Rev. 1975, 75, 731-753.

(47) Hansen, C. M. Hansen Solubility Parameters: A User’s Handbook, 2nd ed.; CRC Press: Boca Raton, FL, 2007.

(48) Duong, D. T.; Walker, B.; Lin, J.; Kim, C.; Love, J.; Purushothaman, B.; Anthony, J. E.; Nguyen, T. Q. Molecular Solubility and Hansen Solubility Parameters for the Analysis of Phase Separation in Bulk Heterojunctions. J. Polym. Sci., Part B: Polym. Phys. 2012, 50, 1405-1413. 
(49) Huang, J. C.; Deanin, R. D. Multicomponent Solubility Parameters of Poly(Vinyl Chloride) and Poly(Tetramethylene Glycol). Fluid Phase Equilib. 2005, 227, 125-133.

(50) Boudouris, B. W.; Ho, V.; Jimison, L. H.; Toney, M. F.; Salleo, A.; Segalman, R. A. Real-Time Observation of Poly(3Alkylthiophene) Crystallization and Correlation with Transient Optoelectronic Proper- ties. Macromolecules 2011, 44, 6653-6658.

(51) Levine, H.; Slade, L. Water as a Plasticizer: Physico-Chemical Aspects of Low-Moisture Polymeric Systems. Water Sci. Rev. 2016, 3, 79-185.

(52) Kjellander, R.; Florin, E. Water Structure and Changes in Thermal Stability of the System Poly (Ethylene Oxide)-water. J. Chem. Soc., Faraday Trans. 1 1981, 77, 2053-2077.

(53) Carlsson, M.; Hallen, D.; Linse, P. Mixing Enthalpy and Phase Separation in a Poly(Propylene Oxide) - Water System. J. Chem. Soc., Faraday Trans. 1995, 91, 2081-2085.

6547

DOI: 10.1021/acs.chemmater.8b05259 Chem. Mater. 2019, 31, 6540-6547 